\title{
Wave Localization Characteristics in the Time Domain
}

\author{
Benjamin White, Ping Sheng, and Zhao Qing Zhang (a) \\ Exxon Research and Engineering Company. Annandale, New Jersey 08801 \\ and \\ George Papanicolaou \\ Courant Institute of Mathematical Sciences, New York University, New York, New York 10012
}

(Received 2 July 1987)

\begin{abstract}
We present an analytical solution to the problem of pulse backscattering from a randomly stratified half-space. It is shown that the power spectrum $\mu$ for the backscattered wave is characterized by a function of $\chi$, where $\chi=($ distance traveled by the pulse at time $\tau) /($ the frequency-dependent localization length). For the matched-impedance and the total-reflection boundary conditions, $\mu$ is given by $\chi /(1+\chi)^{2}$ and $4 \chi$, respectively. Implications for the time-domain measurement of the localization length are discussed.

PACS numbers: 42.20.-y, 03.40.Kf, 43.50.+y, 63.50.+x
\end{abstract}

The phenomenon of wave localization ${ }^{1}$ is usually described in terms of the spatial correlation for a singlefrequency wave, where the exponential decay of the wave envelope is defined as signifying localization. In some recent works, ${ }^{2,3}$ however, we have shown that in the time domain the localization of a pulse exhibits a distinctively different set of characteristics. In particular, results of numerical simulation indicate that in one dimension, the nonstationary power spectrum $\mu$ for the backscattered pulse amplitude is a function of the variable $\chi=c_{0} \tau$ / $l(\omega)$, where $c_{0}$ is the effective-medium speed, $\tau$ the observation time, and $l(\omega)$ the frequency-dependent localization length. By expressing a generic link between the multiple-scattering noise and the localization length, the function $\mu$ demonstrates a clear manifestation of the localization phenomenon in the time domain. In this Letter, we report the first analytical solution to the problem of pulse reflection from a randomly stratified halfspace that leads to an explicit determination of $\mu(\chi)$. The solution is made possible by our taking the "whitenoise" limit, i.e., the correlation length $l_{0}$ of the inhomogeneities is assumed to vanish. However, comparison with numerical simulation data indicates that the analytical result is valid even when $l_{0}$ is finite and large as compared to the wavelength. We offer a plausible reason for the robust nature of the solution and discuss implications for the time-domain measurement of $l(\omega)$.

Consider a medium which is randomly layered in the region $0 \leq x<\infty$, characterized by spatially varying density $\rho(x)$ and bulk modulus $K(x)$, and homogeneous in the region $-\infty<x<0$ with $\rho=\langle\rho(x)\rangle_{\mathrm{av}}=\rho_{0}$ and $K^{-1}=\left\langle K^{-1}(x)\right\rangle_{\mathrm{av}}=K_{0}^{-1}$. The equation governing the propagation of an elastic wave can be written as

$$
\left.\frac{d}{d x}\left(\begin{array}{l}
p \\
v
\end{array}\right)=-\left[\begin{array}{cc}
0 & \rho(x) \\
K^{-1}(x) & 0
\end{array}\right]\right)\left(\begin{array}{l}
\dot{p} \\
\dot{v}
\end{array}\right),
$$

where $p$ denotes pressure, $v$ the displacement velocity, and the overdot denotes time derivative. It should be noted that the propagation of an electromagnetic wave in a stratified medium is governed by an equation which is isomorphic ${ }^{4}$ to Eq. (1). Since the problem is linear, the response of the medium to a pressure pulse is given by

$$
p(\tau)=(2 \pi)^{-1} \int_{-\infty}^{\infty} d \omega \exp (i \omega \tau) f(\omega) R(\omega),
$$

where $f(\omega)$ is the pulse frequency spectrum and $R(\omega)$ the reflection coefficient. The statistics of $p(\tau)$ can be shown to be Gaussian with mean zero. The proof, which is somewhat technical, will be given in a more complete report. ${ }^{5}$ However, given the Gaussian statistics of $p(\tau)$, the knowledge of the power spectrum represents a complete solution to the problem.

The power spectrum of the response, $N(\tau, \omega)$, to a $\delta$ function incident pulse $[f(\omega)=1]$ is related to the time correlation function of $p(\tau)$ :

$$
\begin{aligned}
& \langle p(\tau) p(\tau+t)\rangle \\
& \quad=(2 \pi)^{-1} \int_{-\infty}^{\infty} d \omega \exp [-i \omega t] N(\tau, \omega),
\end{aligned}
$$

where the angular brackets denote configurational averaging, and the approximate stationarity of $p(\tau)$, for small variations in $\tau$, is assumed (this is justified by the final result). From Eq. (2), $N(\tau, \omega)$ can be written as

$$
N(\tau, \omega)=(2 \pi)^{-1} \int_{-\infty}^{\infty} d h \exp (-i h \tau) u(h, \omega)
$$
where

$$
u(h, \omega)=\left\langle R(\omega-h / 2) R^{*}(\omega+h / 2)\right\rangle
$$

is the correlation function of the reflection coefficient at two different frequencies, with $h$ denoting the frequency difference. To calculate $R$ and the correlation function $u(h, \omega)$, we proceed from Eq. (1) by first writing $\rho(x)=\rho_{0}[1+\hat{\rho}(x)], K^{-1}(x)=K_{0}^{-1}[1+\hat{\sigma}(x)]$, where $\hat{\rho}$ 
$(\hat{\sigma})$ denotes the fluctuating part of $\rho\left(K^{-1}\right)$, and then nondimensionalizing all the variables by expressing them in terms of natural units, i.e., $p \rightarrow p / K_{0}, v \rightarrow v / c_{0}$, $x \rightarrow x / l_{0}$, and $\omega \rightarrow \omega l_{0} / c_{0}$, where $c_{0}=\left(K_{0} / \rho_{0}\right)^{1 / 2}$, and $l_{0}$ is the correlation distance of the inhomogeneities. The resulting equation in terms of the right- and lefttravelling-wave amplitudes $A$ and $B$, respectively, is

$$
\frac{d}{d x}\left(\begin{array}{l}
A \\
B
\end{array}\right)=i \omega\left[\begin{array}{cc}
m & -n \exp (-2 i \omega x) \\
n \exp (2 i \omega x) & -m
\end{array}\right]\left(\begin{array}{l}
A \\
B
\end{array}\right),
$$

where we have assumed harmonic time dependence for $p$ and $v, n=\frac{1}{2}(\hat{\rho}-\hat{\sigma}), m=\frac{1}{2}(\hat{\rho}+\hat{\sigma})$, and

$$
\begin{aligned}
& p=\frac{1}{2}[A \exp (i \omega x)+B \exp (-i \omega x)], \\
& v=\frac{1}{2}[A \exp (i \omega x)-B \exp (-i \omega x)] .
\end{aligned}
$$

Defining the reflection coefficient $R=B / A$, we get from Eq. (4) a Riccati equation for $R$ :

$$
d R / d x=i \omega\left[n \exp (2 i \omega x)-2 m R+n R^{2} \exp (-2 i \omega x)\right] .
$$

For a finite slab of length $L$, we have $R(L)=0$, and Eq. (5) is to be integrated backward to $x=0$. As $L \rightarrow \infty$, the boundary condition at $x=L$ can be determined by our noting first that $|R|=1$ because of the localizing nature of the random medium, then writing $R=\exp (i \psi)$ everywhere to obtain a corresponding equation for $\psi$ :

$$
d \psi / d x=2 \omega[n \cos (\psi-2 \omega x)-m] .
$$

Instead of a boundary condition at $x=L$ we now take the statistically stationary solution of Eq. (6). In terms of $\psi$, the function $u(h, \omega)$ can be rewritten as

$$
\begin{aligned}
u(h, \omega) & =\left\langle\exp \left[i\left(\psi_{1}-\psi_{2}\right)\right]\right\rangle \\
& =\int_{0}^{2 \pi} d \bar{\psi} P(\bar{\psi}) \exp (i \bar{\psi}),
\end{aligned}
$$

where $\psi_{1}$ corresponds to frequency $\omega-h / 2$ and $\psi_{2}$ corresponds to frequency $\omega+h / 2, \bar{\psi} \equiv \psi_{1}-\psi_{2}$, and $P(\bar{\psi})$ is the stationary distribution function for $\bar{\psi}$.

In order to calculate $P(\bar{\psi})$ and $u(h, \omega)$, we will take the white-noise limit of $l_{0} \rightarrow 0$. More precisely, the limit process involves the introduction of a dimensionless parameter $l_{0} \omega / c_{0} \equiv \epsilon \ll 1$ and specification that $l_{0}$ is on the order of $\epsilon^{2}$ when measured in terms of a macroscale, which is taken to be 1 . Since $l_{0} \omega / c_{0}=\epsilon$, we get $\omega \rightarrow \omega / \epsilon$ so that the wavelength is on the order of $c_{0}(\omega / \epsilon)^{-1} \sim \epsilon$. On the other hand, $h$ is the frequency scale which determines the correlation and beating between two waves. Since $u(h, \omega)$ is nonzero in only a small range $\Delta h / \omega$ $\sim \epsilon \ll 1$, we require $h \rightarrow h$ under the scaling transformation. By using Eq. (6) to write one equation for $\psi_{1}$ (frequency $\omega-h / 2$ ) and another equation for $\psi_{2}$ (frequency $\omega$ th $/ 2$ ), and by substituting $\omega / \epsilon$ for $\omega$ and making the transformation $\psi_{0}=\frac{1}{2}\left(\psi_{1}+\psi_{2}\right), \bar{\psi}=\psi_{1}-\psi_{2}$, we obtain

$$
\begin{aligned}
& \frac{d \bar{\psi}^{\epsilon}}{d x}=-4 \frac{\omega}{\epsilon} n \sin \left(\psi_{0}^{\epsilon}-2 \frac{\omega}{\epsilon} x\right) \sin \left(\frac{\bar{\psi}^{\epsilon}}{2}+h x\right)-2 h\left[n \cos \left(\psi_{0}^{\epsilon}-2 \frac{\omega}{\epsilon} x\right) \cos \left(h x+\frac{\bar{\psi}^{\epsilon}}{2}\right)-m\right] \\
& \frac{d \psi_{0}^{\epsilon}}{d x}=2 \frac{\omega}{\epsilon}\left[n \cos \left(\psi_{0}^{\epsilon}-2 \frac{\omega}{\epsilon} x\right) \cos \left(h x+\frac{\bar{\psi}^{\epsilon}}{2}\right)-m\right]+h n \sin \left(\psi_{0}^{\epsilon}-2 \frac{\omega}{\epsilon} x\right) \sin \left(h x+\frac{\bar{\psi}^{\epsilon}}{2}\right),
\end{aligned}
$$

where the superscript $\epsilon$ denotes the solution at a given value of $\epsilon$, and the variable dependence of $n\left(x / \epsilon^{2}\right), m\left(x / \epsilon^{2}\right)$ has been suppressed. If we assumed that the random material fluctuations are generated by an ergodic Markov process $q^{\epsilon}(x)=q\left(x / \epsilon^{2}\right)$ in space $\mathbb{R}^{d}$ with an infinitesimal generator $Q_{x}$, then $n$ and $m$ can be written as $n\left(x / \epsilon^{2}\right)=n\left(q\left(x / \epsilon^{2}\right)\right)$ and $m\left(x / \epsilon^{2}\right)=m\left(q\left(x / \epsilon^{2}\right)\right)$. The process $\left(q^{\epsilon}, \bar{\psi}^{\epsilon}, \psi_{0}^{\epsilon}\right) \epsilon \mathbb{R}^{d+2}$ is now jointly Markovian with the infinitesimal generator

$$
L_{x}^{\epsilon}=\frac{1}{\epsilon^{2}} Q_{x}+\frac{d \bar{\psi}^{\epsilon}}{d x} \frac{\partial}{\partial \bar{\psi}^{\epsilon}}+\frac{d \psi_{0}^{\epsilon}}{d x} \frac{\partial}{\partial \psi_{0}^{\epsilon}},
$$

where $d \bar{\psi}^{\epsilon} / d x$ and $d \psi_{0}^{\epsilon} / d x$ are given by Eqs. (8). The problem cast in the form of Eqs. (8) and (9) is now amenable to application of limit theorems of stochastic differential equations. ${ }^{6-9}$ It can be shown that $\left(\bar{\psi}^{\epsilon}, \psi^{\epsilon}\right)$ converges to a limit $\left(\bar{\psi}, \psi_{0}\right)$ as $\epsilon \rightarrow 0$ which is independent of $q$. All the information left over from the $\epsilon^{2}$ scale is captured by the averages of the second moments of $n$ and $m$. By straightforward calculation, the limiting infinitesimal generator is found to be

$$
L_{x}=4 \omega^{2}\left(\left\{\alpha_{m m}+\frac{1}{4} \alpha_{n n}[1+\cos (\bar{\psi}+2 h x)]\right\} \partial^{2} / \partial \psi_{0}^{2}+\alpha_{n n}[1-\cos (\bar{\psi}+2 h x)] \partial^{2} / \partial \bar{\psi}^{2}\right) .
$$

Here

$$
\alpha_{n n}=\int_{0}^{\infty}\langle n(0) n(\xi)\rangle d \xi
$$

and similarly for $\alpha_{m m}$. Note that the coefficients in Eq. (10) do not depend on $\psi_{0}$, so that $\bar{\psi}$ is Markovian by itself. That means that $P(\bar{\psi})$ satisfies the Fokker-Planck

$$
\begin{aligned}
& \text { equation } L_{x}^{*} P=0 \text {, or } \\
& \qquad 2 \omega^{2} \alpha_{n n} \frac{\partial}{\partial z}\left(1+z^{2}\right) \frac{\partial P}{\partial z}-h \frac{\partial}{\partial z}\left(1+z^{2}\right) P=0,
\end{aligned}
$$

where we have made the substitution $z=\cot (\bar{\psi}+2 h x) / 2$ in Eq. (10). From Eq. (12) the distribution function $P$ 
can be solved directly by quadrature, and consequently the function $u(h, \omega)$ may be written as

$$
\begin{aligned}
u(h, \omega) & =u(y) \\
& =y \int_{0}^{\infty} \exp (-\xi y) \frac{\xi}{\xi+i} d \xi, \quad y>0,
\end{aligned}
$$

and $u^{*}(-y)=u(y)$. Here $y=h l(\omega) / c_{0}$, and $l(\omega)$ $\equiv c_{0}\left(\alpha_{n n} \omega^{2}\right)^{-1}$ is identified as the localization length in the white-noise limit. Substitution of Eq. (13) into Eq. (3b) yields, after some elementary integration,

$$
\begin{aligned}
& N(\tau, \omega)=\tau^{-1} \mu(\chi), \\
& \mu(\chi)=\left\{\begin{array}{l}
\chi /(1+\chi)^{2}, \quad \chi \geq 0, \\
0, \quad \chi<0,
\end{array}\right.
\end{aligned}
$$

with $\chi \equiv c_{0} \tau / l(\omega)$. It is noted that $u(y)$ and $\chi^{-1} \mu(\chi)$ are Fourier transforms of each other, and $\mu(\chi)$ satisfies the sum rule that $\chi^{-1} \mu(\chi)$ integrates to 1 , which arises from the requirement that $u(0)=1$.

Equations (13) and (14) are derived with the assumption that the randomly layered half-space is bordered by a homogeneous half-space. We can equally well assume that the random medium interfaces with vacuum, in which case the backscattered wave will be totally returned into the random medium. If one imposes a pressure pulse on the boundary at $\tau=0$ and monitors the medium response in terms of the displacement velocity, then the problem is similar in all respects except that now

$$
u(h, \omega)=\lim _{r \rightarrow 1^{-}}\left\langle\frac{3 r \exp (i \bar{\psi})+1}{1-r \exp (i \bar{\psi})}\right\rangle .
$$

By using the same $P(\psi)$ for averaging and Fourier transforming the result, one gets

$$
\mu(\chi)=4 \chi+\delta(\chi), \quad \chi \geq 0,
$$

where $\delta(\chi)$ denotes the $\delta$ function, and $\mu(\chi)=0$ for $\chi<0$.

To compare our result with numerical simulation data which can extend beyond the white-noise limit, we choose to focus on the quantity $u(y)$ since one can utilize the transfer-matrix technique ${ }^{2}$ for its efficient and accurate determination. The matched-impedance boundary condition is imposed. Three different models of the random medium have been used. In the first one we consider $\rho_{0}=K_{0}=1, \hat{\rho}=0.9 q_{i}(x)$, and $\hat{\sigma}=0.9 q_{j}(x)$, where $q_{i}(x)$ is a random jump process whose value is distributed uniformly in the interval $[-1,1]$ and whose jump interval $l$ follows a probability distribution of $\exp (-l)$. The second model has fixed layer thickness of $l=1, \rho_{0}=8, K_{0}=2$, and $\hat{\rho}=0.05 N_{i}, \hat{\sigma}=0.4 N_{j}$, where $N_{i}$ is a random number with a uniform distribution in $[-1,1]$. The third model is obtained from the first model by the use of an exponential filter for the material parameters $^{10}$ :

$$
m_{3}(x)=\int_{0}^{x} \exp (s-x) m_{1}(s) d s
$$

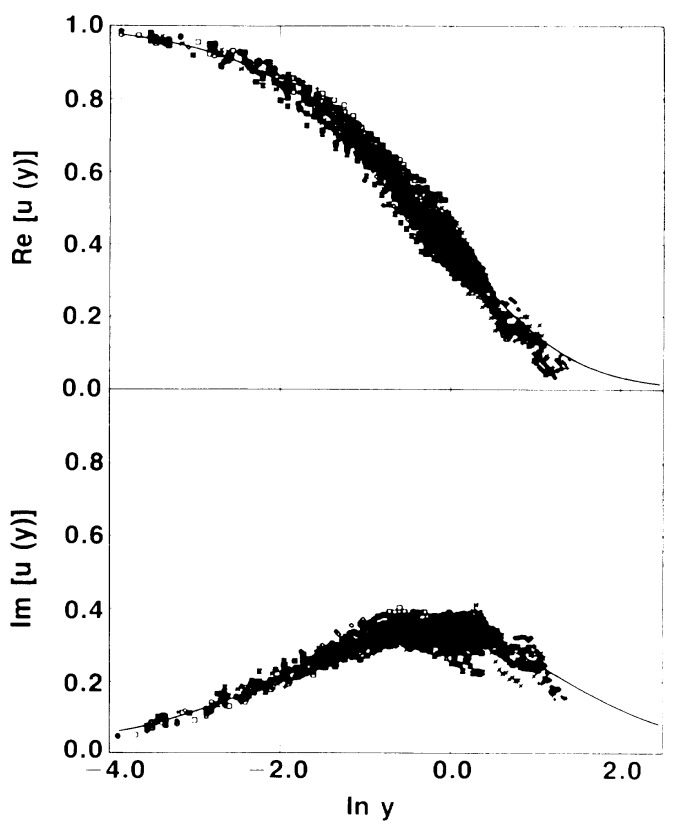

FIG. 1. The real and imaginary parts of $u(y)$ for the matched-impedance case plotted as functions of $\ln y$, where $y=h l(\omega) / c_{0}$. Solid lines denote the theory, and symbols denote the simulation results for a total of 32 different cases involving the three models discussed in the text and different values of $\omega$. Each symbol represents the average over forty configurations.

where $m_{1(3)}$ denotes $\rho$ or $K^{-1}$ for the first (third) model. Since $m_{3}(x)$ is always a continuous function of $x$, the localization behavior of the third model has been shown to differ qualitatively from the other two. ${ }^{10} \mathrm{~A}$ total of 32 different cases, with frequencies ranging from $l_{0} \omega / c_{0}$ $\sim 0.05$ to $l_{0} \omega / c_{0} \sim 10$, have been simulated. In all cases the numerically calculated $l(\omega)$ values have been used in the evaluation of the variable $y=h l(\omega) / c_{0}$. In Fig. 1 we plot all the simulation data for $u(y)$ and compare them with the analytical prediction, Eq. (13). It is seen that despite the fact that the data are from different models and different frequencies, they all show excellent agreement with each other and with the theory. While the independence of $u(y)$ from the different types of model and model parameters is implicit in our derivation and therefore understandable, the validity of the result at all frequency ranges is not anticipated. However, we observe that for classical waves in one dimension, $l(\omega)$ is always bounded below by a fairly large minimum value ${ }^{10}$ even for a medium with very large fluctuations in the material parameters. That means that if we examine the wave attenuation due to multiple scattering, then the dimensionless parameter $Q^{-1}=2 c_{0} / \omega l(\omega)$ will have a maximum value that is $\ll 1$ for all frequencies ( $Q$ is usually defined as the "quality factor"). We speculate that the general condition for the validity of the theory is for $Q^{-1}$ to be small, and that $\omega l_{0} / c_{0} \ll 1$ is just one realization of the general condition. In any case, the compar- 
ison shown in Fig. 1 clearly demonstrates the general applicability of our result.

The knowledge of $\mu(\chi)$ has direct implications for the measurement of $l(\omega)$ in the time domain. In the case of matched-impedance boundary condition, it indicates that on measuring $\tau N(\tau, \omega)$ at a certain frequency window, one should observe a peak at some time $\tau_{0}$ that corresponds to $\chi=1$. Thus, knowing the mean speed of the random medium would yield $l(\omega)=\tau_{0} c_{0}$. If $c_{0}$ is not known, one can still get a relative ratio of the localization lengths for different random media by comparing the peaking times. A curious corollary of our result is that the maximum $\tau N(\tau, \omega)$ value obtained for all onedimensional random systems should be the same, regardless of the magnitude of the material-parameter fluctuations. However, for the medium with smaller randomness one has to wait longer before the maximum $\tau N(\tau, \omega)$ is achieved. For the pressure-release boundary conditions, on the other hand, $N(\tau, \omega)=\tau^{-1} 4 \chi=4 c_{0} /$ $l(\omega)$. That is, the noise spectrum is stationary. This should be obvious since the pulse energy is trapped by a totally reflecting boundary on the one end and by a nonpenetrable random medium on the other. The localization length in this case is directly proportional to the inverse of the noise spectrum and therefore can again be determined directly in the time domain.

In summary, we have obtained an analytic solution to the problem of pulse localization in one dimension and demonstrated its general validity. Application of our approach to the problem of statistical data inversion is presently being pursued.

(a) Present address: Institute of Physics, Academia Sinica, Beijing, People's Republic of China.

${ }^{1}$ Many reviews and references on localization can be found in Percolation, Localization, and Superconductivity, edited by A. M. Goldman and S. A. Wolf, NATO Advanced Study Institute, Series B Vol. 109 (Plenum, New York, 1984).

2P. Sheng, Z. Q. Zhang, B. White, and G. Papanicolaou, Phys. Rev. Lett. 57, 1000 (1986).

${ }^{3}$ R. Burridge, G. Papanicolaou, and B. White, SIAM (Soc. Ind. Appl. Math.) J. Appl. Math. 47, 146 (1987).

${ }^{4}$ With $\epsilon / c \rightarrow \rho, c / \mu \rightarrow K, E \rightarrow v$, and $B \rightarrow p$, where $c$ is the speed of light, $\epsilon$ the dielectric constant, $\mu$ the magnetic permeability, $E$ the transverse electric field, and $B$ the transverse magnetic field.

${ }^{5}$ R. Burridge, G. Papanicolaou, P. Sheng, and B. White, to be published.

${ }^{6}$ G. Papanicolaou, in Studies in Probability, edited by M. Rosenblatt, Mathematical Association of America Studies No. 18 (Mathematical Association of America, Oberlin, $\mathrm{OH}$, 1978), p. 111.

${ }^{7}$ H. J. Kushner, Approximation and Weak Convergence Method for Random Processes, with Applications to Stochastic Systems Theory (M.I.T. Press, Cambridge, MA, 1984).

${ }^{8}$ V. I. Klyatskin, Stochastic Equations and Waves in Random Media (Nauka, Moscow, 1980).

${ }^{9}$ R. Z. Khasminskii, Theory Probab. Its Appl. (Eng. Transl.) 11, 390 (1966).

${ }^{10} \mathrm{P}$. Sheng, B. White, Z. Q. Zhang, and G. Papanicolaou, Phys. Rev. B 34, 4757 (1986). 